\title{
Observations of hard spectrum Unassociated Fermi Objects with MAGIC
}

\section{Konstancja Satalecka ${ }^{* a}$, Simona Paiano ${ }^{b}$, Juan Abel Barrio ${ }^{a}$, Javier Rico ${ }^{c}$ for the MAGIC Collaboration ${ }^{\dagger}$}

E-mail: satalk@gae.ucm.es, spaianolpd.infn.it, barrio@gae.ucm.es, jrico@ifae.es

${ }^{a}$ UCM, Madrid, Spain; ${ }^{b}$ INFN, Padova, Italy; ${ }^{c}$ IFAE, Barcelona, Spain

More than one-third of the sources reported in the 1st and 2nd Fermi catalogs (1FGL and 2FGL) lack a clear association with a known astrophysical source, and are known as Unassociated Fermi Objects (UFOs). We report MAGIC observations of four UFOs selected as MAGIC targets based on their high energy spectral properties (e.g. hardness of the spectrum), as well as on additional multiwavelength information, strongly suggesting a blazar origin for their emission. No significant signal was detected from any of them. In this contribution we present the MAGIC upper limits combined with the collected multiwavelength spectral energy distribution of those sources.

The 34th International Cosmic Ray Conference,

30 July- 6 August, 2015

The Hague, The Netherlands

${ }^{*}$ Speaker.

${ }^{\dagger}$ Full author list at https://wwwmagic.mpp.mpg.de/ 


\section{Introduction}

The First Fermi-LAT catalog (1FGL), published in 2010, contains 1451 high energy $\gamma$-ray sources detected by the LAT instrument in the first 11 months of the science phase of the mission [1]. In 2012 it was followed by the Second Fermi-LAT catalog (2FGL), which spans 24 months of observations and contains 1873 sources [2]. Both catalogs provide, for each of their sources, positional and spectral information, as well as identification or possible associations with sources cataloged at other wavelengths. Although Fermi-LAT has a good angular resolution, a firm identification based on positional coincidence alone is not always feasible: 630 (605) sources in the 1FGL (2FGL) remain unassociated. Similar numbers can be given for the Third Fermi-LAT cata$\log$ (3FGL [3]): 1009 out of 3033 sources are UFOs.

Therefore more than one-third of the reported sources lack a clear association with a known astrophysical source, and are known as Unassociated Fermi Objects (UFOs). This makes an important component of the $\gamma$-ray sky, and may hide new classes of AGN, dark matter clump candidates, or even new and unexpected high-energy phenomena. Starting in 2010, the MAGIC collaboration developed a program to search for interesting targets among those enigmatic objects.

\section{Selection Criteria}

Search criteria were defined in order to identify possible MAGIC targets among UFO population. Altough the provided information slightly changed from 1FGL (see [4]) to 2FGL [5], the underlying selection criteria remained common, as follows:

- To lay outside the Galactic Plane. Source association in a very crowded environment, such as the Galactic plane, is more difficult. UFOs with galactic latitudes $|b|<10^{\circ}$ were discarded.

- To be non-variable. A constant photon flux guarantees a more reliable extrapolation into the MAGIC energy range. Sources whose variability index surpasses $<1 \%$ likelihood of being a steady source limit were discarded.

- To be detected and have a hard spectrum at $\mathrm{GeV}$ energies. We require: a power-law with spectral index $>-2.5$ and integral photon flux in the 10 to $100 \mathrm{GeV}$ energy bin detected with Test Statistics ${ }^{1}$ of: $\sqrt{T S}>4.0$.

\subsection{Fermi high energy $\gamma$-ray data}

After the selection based on the catalogs information we checked the robustness of FermiLAT measurement at the $10 \mathrm{GeV}-100 \mathrm{GeV}$ energy band and the validity of the extrapolation of Fermi-LAT spectra to the IACT energy range.

Fermi data were studied for all the considered sources using the latest version of the Fermi ScienceTools [6]. The best suited event selection quality cuts for off-plane point source analysis were applied by means of the gt select tool. Additionally, a maximum zenith angle cut of $105^{\circ}$ was applied and the latest instrument response functions were used. Regarding the time

\footnotetext{
${ }^{1}$ The square root of TS approximately corresponds to the significance of detection of the source.
} 
selection, performed with the gtmktime, only good time intervals were considered. On top of that, photons arriving when the satellite was crossing the South Atlantic Anomaly were discarded as well as those recorded at a rocking angle greater than $45^{\circ}$. Regions of interest based zenith angle cuts were also applied. A more complete description of Fermi-LAT data analysis can be found in [7].

Out of this study, we extracted the total number of High Energy photons (HE, $E_{\gamma}>10 \mathrm{GeV}$ ) observed by Fermi-LAT in a conservative circular region corresponding to the Fermi-LAT Point Spread Function radius at $10 \mathrm{GeV}$ for $68 \%$ containment, centred on each of the potential targets $\left(0.25^{\circ}\right)$. The contribution from diffuse HE $\gamma$-ray background in each source position was estimated from actual data to be almost negligible.

\subsection{MAGIC Detection Prospects}

In order to estimate the detection prospects of Fermi UFO for the MAGIC telescopes, the VHE UFO spectra were directly extrapolated from the Fermi catalogs, assuming a power law. In the IACT context a source is considered as detected, if the number of observed excess events is more than 5 standard deviations $(5 \sigma)$ away from the number of background events. The significance of the measurement is expected to scale with the square root of the exposure time. We used Eq. 17 from $\mathrm{Li}$ and $\mathrm{Ma}$ [8] to estimate the time that MAGIC would need to detect a given source with a significance of $5 \sigma$. The MAGIC background rate and effective area required for this computation were taken from [9].

Finally, sources were ordered according to the estimated detection time. In case of similar estimated detection times, sources with a larger Fermi HE photons population were given higher priority.

Out of these sorting criteria, and imposing good observational conditions from the MAGIC telescopes latitude $\left(\lesssim 45^{\circ}\right)$, two sources were selected out of the 1FGL catalog: 1FGL J2347.3+0710 and 1FGL J0338.8+1313 hereafter for simplicity UFO-I, and UFO-II. Later, two source were selected out of the 2FGL catalog: 2FGL J1410.4+7411 (UFO-III) and 2FGL J1511-0513 (UFO-IV).

Table 1 shows the Fermi-LAT spectral features for the four above mentioned sources, along with the number of photons with energies above $10 \mathrm{GeV}$ and $50 \mathrm{GeV}$, as well as the TS value between $10 \mathrm{GeV}$ and $100 \mathrm{GeV}$.

\begin{tabular}{llllllll}
\hline Source name & $\begin{array}{l}\text { Naming } \\
\text { convention }\end{array}$ & $\begin{array}{l}\mathrm{E} \\
{[\mathrm{GeV}]}\end{array}$ & $\begin{array}{c}F_{0} \\
{\left[1 / \mathrm{cm}^{2} / \mathrm{MeV} / \mathrm{s}\right]}\end{array}$ & $\begin{array}{l}\text { Spectral } \\
\text { index }\end{array}$ & $\begin{array}{l}\sqrt{T S} \\
{[\% \text { C.U.] }}\end{array}$ & $N_{\gamma, 1}$ & $N_{\gamma, 2}$ \\
\hline 1FGL J2347.3+0710 & UFO-I & 8.3 & $(1.1 \pm 0.4) \cdot 10^{-14}$ & $1.5 \pm 0.35$ & 4.5 & 2 & 1 \\
1FGL J0338.8+1313 & UFO-II & 4.5 & $(4.1 \pm 1.2) \cdot 10^{-14}$ & $1.8 \pm 0.2$ & 3.6 & 3 & 1 \\
2FGL J1410.4+7411 & UFO-III & 2.3 & $(1.2 \pm 0.2) \cdot 10^{-13}$ & $1.9 \pm 0.2$ & 4.7 & 12 & - \\
2FGL J1511.8-0513 & UFO-IV & 2.1 & $(2.9 \pm 0.5) \cdot 10^{-13}$ & $2.22 \pm 0.12$ & 3.8 & 5 & - \\
\hline
\end{tabular}

Table 1: Spectral characteristics of the selected UFOs. $2^{\text {nd }}$ column: source naming convention, as explained in the text; $3^{\text {rd }}$ column: Pivot Energy in GeV; $4^{\text {th }}$ column: Differential photon flux at the Pivot Energy in $1 / \mathrm{cm}^{2} / \mathrm{MeV} / \mathrm{s} ; 5^{\text {th }}$ column: Spectral Index; $6^{\text {th }}$ column: square root of the Test Statistic between 10 and $100 \mathrm{GeV} ; 7^{\text {th }}$ column: number of photons with energies between $10 \mathrm{GeV}$ and $50 \mathrm{GeV} ; 8^{\text {th }}$ column: number of photons with energies greater than $50 \mathrm{GeV}$. 


\section{MAGIC Observations}

MAGIC consists of a system of two $17 \mathrm{~m}$ Imaging Atmospheric Cerenkov telescopes operating in stereoscopic mode since fall 2009 at the Canary Island of La Palma $\left(28.8^{\circ} \mathrm{N}, 17.8^{\circ} \mathrm{W}, 2200\right.$ $\mathrm{m}$ a.s.l.). The observations of UFO-I and UFO-II took place between October 2010 and June 2011, with the initial MAGIC hardware stereoscopic configuration [9]. We collected $8.3 \mathrm{~h}$ and $11 \mathrm{~h}$ of good data for UFO-I and UFO-II respectively. For these observations, the telescopes were pointing at the $1 \mathrm{FGL}$ catalog positions, which were the only ones available at that time. The observations of UFO-III and UFO-IV, as well as a large part of UFO-I observations were performed after December 2012, when an important hardware upgrade took place [10]. For this last observation of UFO-I and UFO-III the telescopes were pointing at the more precise 2FGL catalog positions, while for UFO-IV at its X-ray counterpart: 1SWXRT J151148-051347. We collected $20 \mathrm{~h}, 15 \mathrm{~h}$ and $17 \mathrm{~h}$ of good data for UFO-I, UFO-III and UFO-IV respectively.

All sources have been observed by MAGIC during dark night in false tracking mode (wobble mode) [11], under the lowest zenith angle range possible, obtaining low energy threshold of $150 \mathrm{GeV}$. Only $U F O-I I I$ was observed with zenith angles $45-47^{\circ}$ which resulted in higher analysis threshold of $200 \mathrm{GeV}$. Data were analyzed in the MARS analysis framework by means of the standard stereoscopic analysis routines as described in [9]. In addition, the whole data set was subjected to a quality selection process based on weather and hardware conditions. As a final cross-check of the analysis framework, contemporaneous Crab Nebula data were also used to verify the proper performance of the telescopes and analysis routines.

\subsection{MAGIC data analysis}

The analysis of the data corresponding to each of the four sources did not reveal any significant VHE signal in either night-by-night or cumulative analyses.

Given the uncertainty of the source nominal positions in Fermi-LAT catalogs, a VHE $\gamma$-ray sky map is required in order to discard the possibility that the source may be slightly displaced from the catalog coordinates. Consequently, TS sky maps were produced (see Fig. 1, left column). They do not present any structure compatible with a point-like source, with the significance distributions being consistent with background fluctuations.

\subsection{Results}

Since no VHE signal was confirmed, upper limits to the source emission were computed. The upper limits to the number of signal events were obtained using the Rolke method [13], assuming a systematic error on the gamma-ray detection efficiency of $30 \%$ which accounts for the energy estimation and effective area calculation. The considered confidence level (CL) was 95\%. The upper limits on the differential flux, were calculated assuming a spectral index $\Gamma=2.0$ and the 3FGL catalog source position.

In order to fully profit from the data collected during UFO-I observations we decided to stack the data from the two years and obtain more stringent limits on the VHE $\gamma$-ray flux. In the upper limit calculations we used an average likelihood method. In this method, we summed ON and OFF events from all data samples, as well as their partial observation times. The total effective area was 
defined as an average of the effective areas of all analysed samples, weighted by the observation time of each sample.

The middle column of Fig. 1 shows the extrapolated spectra, as reported in 3FGL, for each of the four sources from $10 \mathrm{GeV}$ to $10 \mathrm{TeV}$ along with the MAGIC differential flux upper limits. As can be seen, the extrapolations of UFO-I and UFO-II power-law spectra above $380 \mathrm{GeV}$ and 150 $\mathrm{GeV}$ respectively were excluded at the $95 \% \mathrm{CL}$.

\section{Dedicated search for UFO counterparts}

An extensive and independent search for possible associations was performed for each selected UFO. We performed dedicated analyses of public Swift-XRT data by means of the Swift-XRT data products generator [17]. X-ray band sky maps were created for all observed UFOs in a search for new and non-cataloged sources. If such a source was found inside the 3FGL catalog 95\% error region, an interactive on-line analysis of Swift-XRT data was performed in order to determine its position and error box (of order of some arcsecs) and to extract the energy spectrum in the 0.3$10 \mathrm{keV}$ band. On the XRT sky map, we then superimposed different astronomical catalogs or surveys. If other sources observed at different wavelength were positionally coincident with this X-ray source, we combined all information through the SED Builder tool of the ASDC archive. Assuming that these sources are the same object observed at different wavelengths, we obtain a SED with the most likely set of counterparts for each UFO.

Finally we estimated the probability, that the SED of a given UFO is that of a conventional blazar, using a AGN SED template tool [19]. This tool uses archival multiwavelength data to create SED templates of different AGN types (HBL, IBL, LBL, FSRQ). Then the UFO SEDs (the $v L(v)$ luminosity in erg/sec versus the photon frequency) are compared to the templates, assuming different values of redshift that span from 0.05 to 2.0, and the most likely AGN type and redshift are estimated for each source using the Minimum Average Distance and $\chi 2$ methods.

\section{Discussion}

UFO-I. As can be seen from Fig. 1, an extrapolation of 3FGL J2346.7+0705 power-law spectrum above $380 \mathrm{GeV}$ is excluded at the $95 \% \mathrm{CL}$. In the 3FGL catalogue this source is reported as active galaxy of uncertain type and associated with the X-ray source proposed in [19]. Redshift of this source is unknown. According to the SED template tool, the SED of this object is compatible with an LBL of $\mathrm{z} \sim 0.2$ (Fig. 1, right column).

UFO-II. The 3FGL catalog indicates a slightly harder spectral index $\left(\alpha_{3 F G L}=1.70 \pm 0.10\right)$ than the one given by $1 \mathrm{FGL}\left(\alpha_{1 F G L}=1.78 \pm 0.22\right)$, but an extrapolation of 3FGL catalog powerlaw spectrum above $150 \mathrm{GeV}$ can be clearly excluded. This fact suggests a possible curvature or cut-off in the spectrum. In July 2012, this source was observed by Swift-XRT. Our dedicated X-ray analysis of this observation shows that there is a point source with a $0.0468 \mathrm{cts} / \mathrm{s}$ count rate in the 0.3-10 keV energy range within the Fermi-LAT 2FGL catalog error contour and coincident with the 3FGL catalog position, as shown in sky map in the left column of Fig. 1. Using the SED template tool, we can derive for 3FGL J0338.2+1306 an HBL-like nature, although the redshift estimation 
is degenerated. Taking into account the curvature above $150 \mathrm{GeV}$ due to Extragalactic Background Light (EBL) extinction, this source should be located at a high redshift (Fig.1, right column) [18]).

UFO-III As can be seen from the middle column of Fig. 1, an extrapolation of 3FGL J1410.4+7411 catalog power-law spectrum cannot be excluded by the present MAGIC upper limits. 3FGL catalog still classifies this source as unassociated. Swift, observations performed in 2014 (after the construction of the 3FGL catalogue), found a weak X-ray source inside the, significantly improved, 3FGL error box of this source. The MWL SED template analysis suggests that this object could be a high redshift HBL (Fig.1, right column).

UFO-IV The present MAGIC upper limits cannot exclude the 3FGL J1511-0513 catalog power-law spectrum. In the 3FGL catalogue this object is an AGN of uncertain type associated to the radio source NVSS J151148-051345. From the Swift observations in 2010 the X-ray counterpart is 1 SWXRT J151148-051347 which X-ray spectrum is determined from 0.3 to $2.0 \mathrm{keV}$. The SED template tool indicates that the MWL SED of this object is compatible with an HBL located to $\mathrm{z} \sim 0.04$. (Fig.1, right column).

\section{Conclusions and outlook}

A dedicated search designed to select possible emitters of VHE gamma rays out of the FermiLAT 1FGL and 2FGL catalogs has been presented. After studying the prospects of detection for each of these candidates, the best four candidates were observed by the MAGIC Telescopes. With the publication of the 3FGL catalog, the obtained results for the 1FGL and 2FGL candidates were revised taking into account the improved positional and spectral reconstruction obtained from the Fermi-LAT instrument.

No VHE $\gamma$-ray signal was detected from any of the sources observed, and therefore upper limits to their differential fluxes were obtained. In particular, for the case of 3FGL J0338.3+1306 and 3FGL J2347.3+0710, a direct extrapolation of the Fermi-LAT spectrum above $150 \mathrm{GeV}$ and $400 \mathrm{GeV}$ can be ruled-out, and some kind of spectral cut-off must take place below that energy. On the contrary, for 3FGL J1410.4+7411 and 3FGL J1511-0513 the MAGIC upper limits are not so stringent.

For each UFO we collected MWL data available through the Swift and ASDC data bases. Using these data we tested the hypotheses that those sources are AGN located at certain redshifts. For each of the sources (apart from 3FGL J0338.2+1306) we propose a unique AGN classification and a redshift. The proposed values need an optical confirmation.

\section{Acknowledgments}

We would like to thank the Instituto de AstrofŠsica de Canarias for the excellent working conditions at the Observatorio del Roque de los Muchachos in La Palma. The financial support of the German BMBF and MPG, the Italian INFN and INAF, the Swiss National Fund SNF, the ERDF under the Spanish MINECO (FPA2012-39502), and the Japanese JSPS and MEXT is gratefully acknowledged. This work was also supported by the Centro de Excelencia Severo Ochoa SEV-2012-0234, CPAN CSD2007-00042, and MultiDark CSD2009-00064 projects of the Spanish Consolider-Ingenio 2010 programme, by grant 268740 of the Academy of Finland, by the Croatian Science Foundation (HrZZ) Project 
09/176 and the University of Rijeka Project 13.12.1.3.02, by the DFG Collaborative Research Centers SFB823/C4 and SFB876/C3, and by the Polish MNiSzW grant 745/N-HESS-MAGIC/2010/0.

\section{References}

[1] Fermi-LAT Collaboration, A. A. Abdo et al., ApJS 188 (2010) 405-436 [arXiv:1002.2280]

[2] P. L. Nolan, A. A. Abdo, et al., ApJS 199 (2012) 31 [arXiv:1108.1435]

[3] Fermi-LAT Collaboration, M. Ackermann et al., accepted for publication in Astrophysical Journal Supplement Series, 2015 [arXiv: 1501.02003]

[4] D. Nieto et al., III Fermi-LAT Symposium, Rome (2011) [arXiv:1110.4744]

[5] D. Nieto et. al., Search for UFOs in 2FGL, Submitted to Physics of the Dark Universe

[6] NASA, http://fermi.gsfc.nasa.gov/ssc/data/analysis/documentation/

[7] NASA, http://fermi.gsfc.nasa.gov/ssc/data/analysis/documentation/Cicerone/

[8] T.-P. Li and Y.-Q. Ma, ApJ 272 (1983) 317-324

[9] MAGIC Collaboration, J. Aleksic et. al., Astropart.Phys. 35 (2012) 7 [arXiv:1108.1477]

[10] MAGIC collaboration, J. Aleksic et al., submitted to Astropart. Phys. (2015)

[11] V. P. Fomin et al., Astropart.Phys. 2 (1994) 137

[12] MAGIC Collaboration, R. Zanin et. al., 33rd ICRC, Rio de Janeiro, Brazil (2013)

[13] W. A. Rolke et al., Nucl.Instrum.Meth. A551 (2005) 493-503, [physics/0403059]

[14] MAGIC Collaboration, J. Albert et. al., ApJ 674 (2008) 1037-1055, [astro-ph/0705.3244]

[15] ASDC: ASI Science Data Center, http://www.asdc.asi.it/, 2011

[16] Y. Takeuchi et al., ApJS 208 (2013) 25, [arXiv:1307.5581]

[17] SWIFT, http://www.swift.ac.uk/user_objects/

[18] R. C. Gilmore et al., MNRAS 422 (2012) 4 [arXiv:1104.0671]

[19] Paiano et al., A New Method for the Identification and Characterization of Unidentified Fermi Objects with blazars, in preparation (submitted to MRAS). 

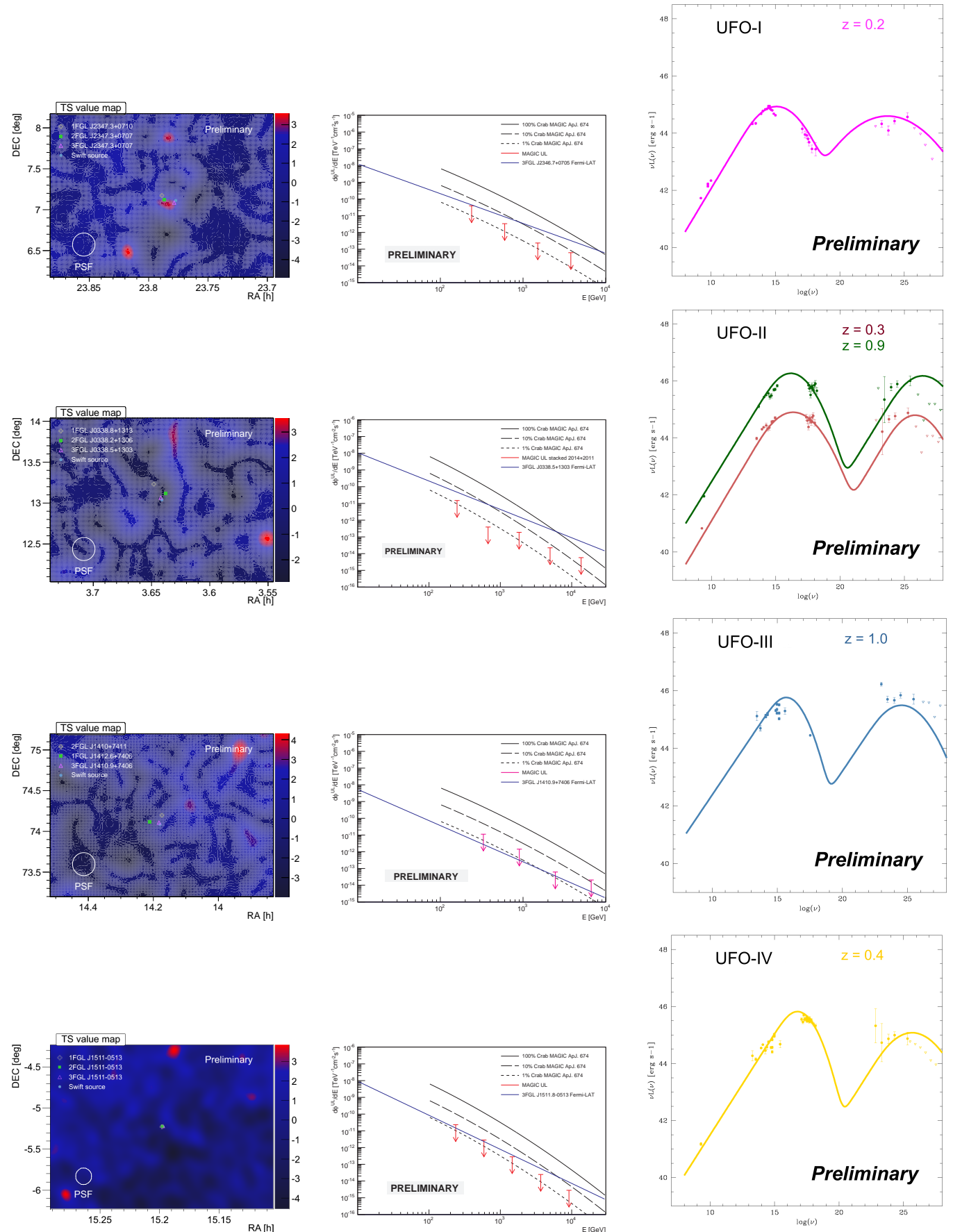

Figure 1: Left column: MAGIC sky maps. The significance distributions are consistent with background fluctuations. The positions of the Fermi sources for 1FGL, 2FGL and 3FGL are shown, as well as the bright X-ray sources in the MAGIC Field of View. The white circle depicts MAGIC PSF for the given energy range. Middle column: MAGIC differential flux upper limits $d \Phi^{U L} / d E$ (red). MAGIC Crab Nebula spectrum [14] (black) is depicted as reference. The extrapolation of the 3FGL catalog Fermi-LAT differential spectrum is also shown (blue). Right column: Spectral luminosity points of the four UFOs observed by MAGIC for different assumed redshift (from 0.05 to 2.0), compared to the blazar SED templates (Figures adapted from Paiano+2015). All plots are arranged from UFO-I (top) to UFO-IV (bottom). 